\title{
Genus Zero Graph Segmentation: Estimation of Intracranial Volume
}

Rasmus R. Jensen ${ }^{1}$, Signe S. Thorup ${ }^{1}$, Rasmus R. Paulsen ${ }^{1}$, Tron A. Darvann ${ }^{2,3}$, Nuno V. Hermann ${ }^{2,4}$, Per Larsen ${ }^{2}$, Sven Kreiborg ${ }^{2,4,5}$, and Rasmus Larsen ${ }^{1}$

${ }^{1}$ DTU Compute, Technical University of Denmark, Lyngby, Denmark

2 3D Craniofacial Image Research Laboratory,

University of Copenhagen, Copenhagen, Denmark

3 Dept. of Oral and Maxillofacial Surgery, Copenhagen University Hospital, Copenhagen, Denmark

4 Pediatric Dentistry and Clinical Genetics, University of Copenhagen, Denmark

5 Dept. of Clinical Genetics, Copenhagen University Hospital, Copenhagen, Denmark

\begin{abstract}
The intracranial volume (ICV) in children with premature fusion of one or more sutures in the calvaria is of interest due to the risk of increased intracranial pressure. Challenges for automatic estimation of ICV include holes in the skull e.g. the foramen magnum and fontanelles. In this paper, we present a fully automatic 3D graph-based method for segmentation of the ICV in non-contrast CT scans. We reformulate the ICV segmentation problem as an optimal genus 0 segmentation problem in a volumetric graph. The graph is the result of a volumetric spherical subsample from the data connected using Delaunay tetrahedralisation. A Markov Random Field is constructed on the graph with probabilities learned from an Expectation Maximisation algorithm matching a Mixture of Gaussians to the data. Results are compared to manual segmentations performed by an expert. We have achieved very high Dice scores ranging from $98.14 \%$ to $99.00 \%$, while volume deviation from the manual segmentation ranges from $0.7 \%-3.7 \%$. The Hausdorff distance, which shows the maximum error from automatic to manual segmentation ranges, from $4.73-9.81 \mathrm{~mm}$. Since this is sensitive to single error, we have also found the 95\% Hausdorff distance, which ranges from 1.10$3.65 \mathrm{~mm}$. The proposed method is expected to perform well for other volumetric segmentations.
\end{abstract}

Keywords: Intracranial volume, CT, craniosynostosis, graph cut, segmentation.

\section{Introduction}

Unicoronal synostosis (UCS) is a congenital craniofacial malformation characterized by the premature fusion of one of the coronal sutures, potentially leading to asymmetric head shape, craniofacial growth disturbances, increased intracranial pressure and developmental delays. Computed Tomography (CT) scanning 
is usually performed to confirm the diagnosis and to facilitate surgical treatment planning. The intracranial volume (ICV) in children with premature fusion of one or more sutures in the calvaria may become reduced, leading to risk of increased intracranial pressure [1]. Challenges for automatic estimation of ICV include holes in the skull in newborns (the fontanelles), but also holes in the cranial base (e.g. the foramen magnum and other foramina, fissures and synchondroses). The main contribution of our work is a fast and fully automatic method for segmentation and estimation of the ICV in CT scans of children with craniosynostosis. The method is based on the construction of a volumetric graph description of the skull volume using tetrahedralization followed by a graph cut forced to robustly perform a genus 0 segmentation. Validation is carried out by comparing the automatic segmentation model to a semi-automated model.

\section{Brief Review of the Previous Research}

Current work on automatic ICV1 estimation has focused on Magnetic Resonance Imaging (MRI) volumes [2-4]. However, these methods are not well suited for ICV estimation in craniosynostotic cases due to the limited bone-tissue contrast in MRI. In the case of craniosynostosis, the best contrast of the cranial bones, e.g. for diagnosis and surgery planning, is obtained from CT scans. Furthermore, standard methods often use atlases based on a normal population, which may lead to a bias in the estimation of the ICV in craniosynostotic cases. The current standard for ICV estimation from CT is a manual method based on thresholding followed by a seed-growing algorithm. The challenge of this method is the need for manual editing in the various foramina in the skull base as well as in regions where craniosynostosis or lacking suture fusion have caused gaps between the cranial bones [5 5 .

Anatomical segmentation such as the segmentation of the ICV in medical images is addressed in the literature by a series of approaches. In [8], deformable template matching is applied in a Bayesian setting; in [9], deformable surface models are proposed using a graph cut approach; and in [10], a multiclass Markov Random Field (MRF) is used for voxel classification. In the latter case it is interesting that, for two-class models, global optimal segmentation can be obtained using a graph-cut-based approach [11]. In this work we propose a two class segmentation of the ICV, where the classes (inside and outside) are modeled as mixtures of Gaussians. In addition to a label prior, we use a gradientdependent interaction term. Moreover, we employ a tetrahedralization of a spherical equidistant sample distribution leading to a graph. The graph has dedicated outside and inside nodes, which robustly forces the graph segmentation to be of genus 0 .

\footnotetext{
${ }^{1}$ In MRI they often estimate the total intracranial volume (TIV).
} 


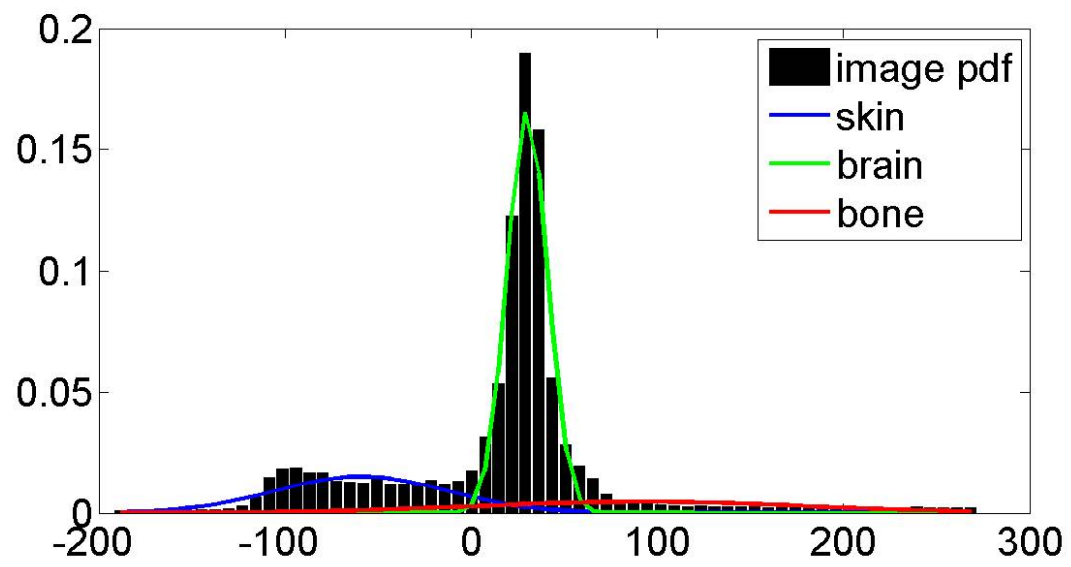

Fig. 1. A mixture of Gaussians shown on the intensity histogram of a CT scan

\section{Approach}

The data consist of pre-surgical CT head scans of 15 children diagnosed with UCS (either left- or right-sided). Age ranged from 6 to 18 months. All scans were acquired at Copenhagen University Hospital, Rigshospitalet, except for one which was acquired at Helsinki University Central Hospital. Because of the UCS and the different age the data set is not homogeneous. All scans were obtained at $512 \times 512$ pixels in-plane size and a complete volume consists of between 167 and 350 slices.

The aim of the method is to create a volumetric segmentation that follows the transition between brain matter and bone, while also closing holes in the bone structure. As the intensities of the CT scans vary, we fit a mixture of Gaussians to each individual scan. The mixture of Gaussians is carried out using expectation maximization and results in three normal distributions describing: skin, brain matter and bone (see Fig. 1). Skin and bone have higher variance compared to brain tissue, which is used to classify the distributions unsupervised. Brain matter is by far the dominant, but also that with the least variance. Using the probability density function, where $v$ is a sample value, we define the following two probabilities: $\mathrm{p}(v \mid x=\mathrm{ICV})=\operatorname{pdf}_{\text {brain }}$ and $\mathrm{p}(v \mid x \neq \mathrm{ICV})=$ $\mathrm{pdf}_{\text {skin }}+\mathrm{pdf}_{\text {bone }}$. Generally, the brain matter distribution fits well to the data, while the other two tissues just stay below and above both with a wider standard deviation. Using only the mixture of Gaussians to classify brain-tissue and nonbrain tissue would lead to misclassification as the distributions are crude, while the proposed method is insensitive to this.

Before the segmentation, the volumes were interpolated in the slice-wise direction to create isotropic voxels and ensure a regular sampling. A graph is created on sampling points in the volumes. The sampling points are found using a spherical volume of quasi-equidistantly distributed nodes. The nodes are distributed 

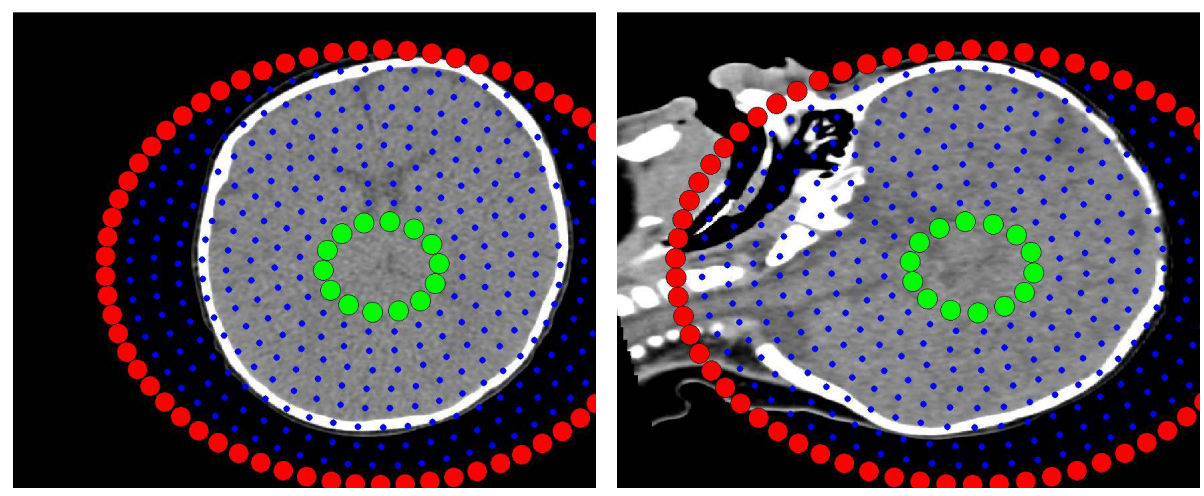

Fig. 2. Examples of distribution of sample points in the transverse and sagittal planes. The sample density is much higher in the actual application with a voxel distance of two between sample points. The green nodes show the inner sphere which is forced to be part of the ICV, while the red nodes shows the outer sphere which is forced to be outside the ICV. The slices are contrast enhanced based on the mixtures of Gaussians.

on the surfaces of concentric spheres, where the differences between their radii are equal to the spacing between their longitudes. Similarly, points on the longitudes are distributed with this spacing. This suggested sampling approach has two benefits: the sampling density can easily be changed for different resolutions, and it also removes the over/under-sampling problem of a spoke-like graph directed from the center and out. The sample volume is centered in the middle of the calvaria and created such that it covers the entire skull. In the center of the graph, we leave a small empty sphere, which will be used to clamp the inside of the graph to the ICV (see Fig. 2). The spherical graph is centered automatically by summing the voxel-wise probability of brain matter given the distribution prior. We find the coordinates of voxels with higher probability than $\frac{2}{3}$ of the maximum summation of the sagittal, coronal and transverse planes, respectively. The center is found as the median of these coordinates, while the radius of the sphere is found as 2.5 times the maximum interquartile range in the sagittal and coronal planes only. Fig. 2 illustrates the sample point distribution in the transverse and sagittal planes. For the actual ICV estimation we have used a much higher sample density, using an even voxel distance of two between sample points. A robust way of connecting each of the sample points to the immediate spatial neighborhood in a highly connected graph can be achieved by Delaunay tetrahedralization [12]. As this approach produces doublets of edges represented by several adjacent tetrahedra the connectivity has to be cleaned up such that edges are represented only once. On the graph with index set $\mathcal{I}$, we define the following MRF, which is solved using graph cuts [11]:

$$
E(\mathbf{x})=\sum_{i \in \mathcal{I}}\left(\Phi\left(v_{i} \mid x_{i}\right)+\sum_{j \in N_{i}}\left(\lambda\left(x_{i}, x_{j}\right)+\psi\left(x_{i}, x_{j}\right)\right)\right)
$$



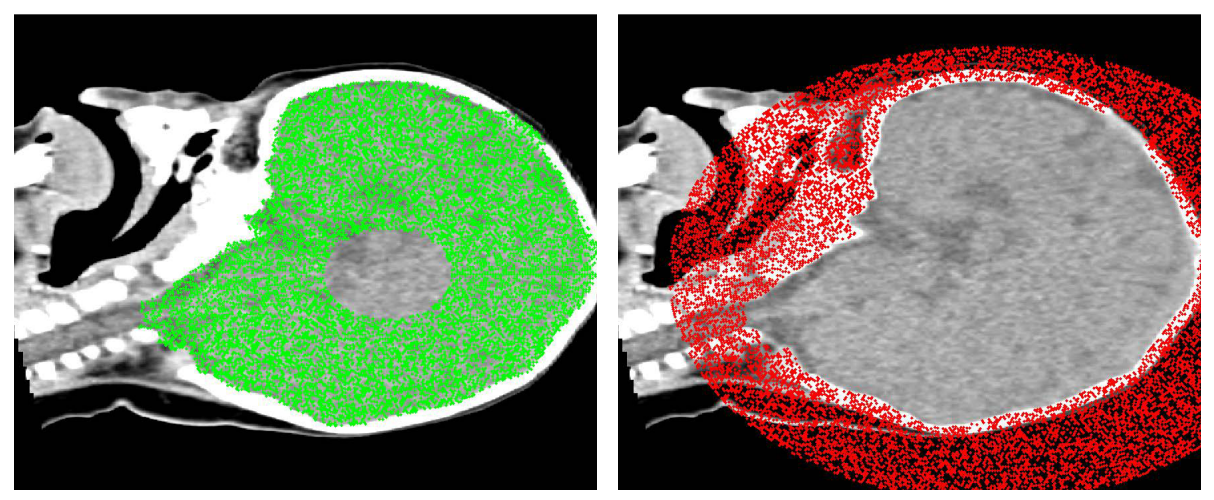

Fig. 3. The result of the segmentation between $I C V$ and outside $I C V$ shown in the sagittal plane. The slices are contrast enhanced based on the mixtures of Gaussians.

$\Phi\left(v_{i} \mid x_{i}\right)=-\log \mathrm{p}\left(v_{i} \mid x_{i}\right)$ defines a log-likelihood function. The function returns high values for low probabilities and vice versa. The outer and inner sphere log-likelihood values are clamped as follows:

$$
\begin{aligned}
& \Phi\left(i \in \text { outer } \mid x_{i}\right)=\left\{\begin{array}{cc}
\infty & x_{i} \neq \mathrm{ICV} \\
0 & x_{i}=\mathrm{ICV} \\
0 & x_{i} \neq \mathrm{ICV} \\
\infty & x_{i}=\mathrm{ICV}
\end{array}\right.
\end{aligned}
$$

$N_{i}$ denotes the neighborhood of the $i$ 'th voxel and has terms defined as:

$$
\lambda\left(x_{i}, x_{j}\right)+\psi\left(x_{i}, x_{j}\right)=\left\{\begin{array}{cl}
K_{\nabla} e^{-\left|\nabla \mathrm{f}\left(x_{i}, x_{j}\right)\right|}+K_{i j} & x_{i} \neq x_{j} \\
0 & x_{i}=x_{j}
\end{array}\right.
$$

Where $\left|\nabla \mathrm{f}\left(x_{i}, x_{j}\right)\right|$ is the absolute gradient; $K_{\nabla}$ controls the power of the gradient term, while $K_{i j}$ is a general smoothness prior. With the outside sphere clamped as outside $I C V$ and the inside sphere clamped as $I C V$, a setting of $K_{\nabla}$ and $K_{i j}$ exists for which the resulting graph cut will only be on the inner edge of the skull, close the holes (e.g. fontanelles, optic canals, foramen magnum, and other foramina), and it will produce a segmentation of genus 0 . We achieved our results with $K_{\nabla}$ being of the same magnitude as $\max \Phi\left(v_{i} \mid x_{i}=\mathrm{ICV}\right), i \in I$ and $K_{i j} \frac{1}{100}$ of that. We found the results to be rather insensitive to fine tuning of $K_{\nabla}$ and $K_{i j}$. The result of a segmentation is shown in Fig. 3. With a segmentation of the graph, the volume can be estimated using the tetrahedralization of the sample points contained in the cut.

\section{Results and Discussion}

Ground truth was made by expert manual segmentations using a semi-automatic, slice-wise method based on a seed-growing algorithm incorporated in Analyze ${ }^{\mathrm{TM}}$ 

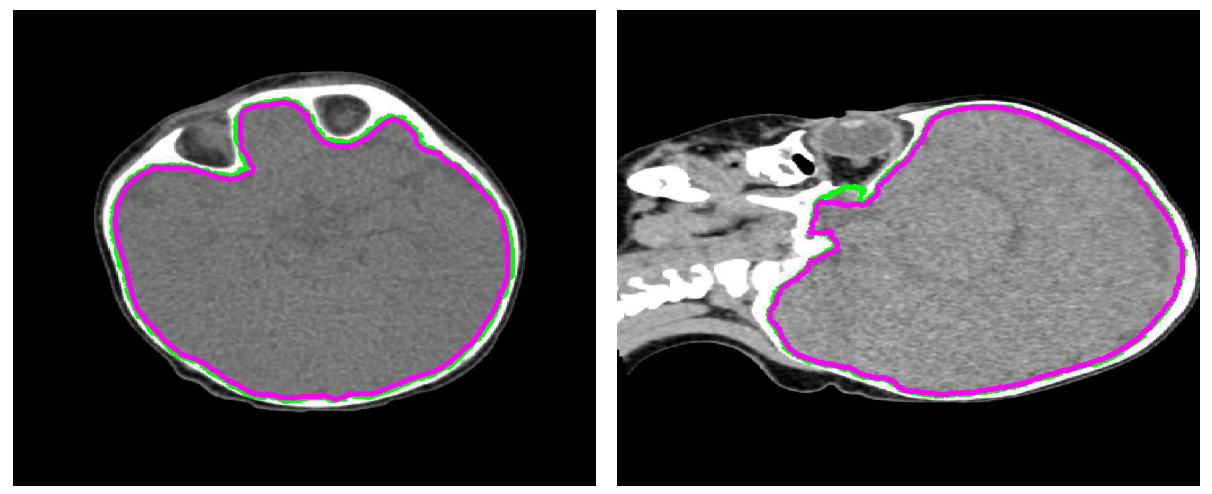

Fig. 4. Examples of the manual (magenta) and automatic segmentation (green) shown on a transversal (Patient \#8) and sagittal slice (Patient \#11), respectively. The slices are contrast enhanced based on the mixtures of Gaussians.

(BIR Research Lab, Mayo Clinic, Rochester, MN, USA). This method requires a user-specified intensity threshold and manual editing.

As gaps and small fractures are present in the data, the semi-automatic segmentation algorithm often breaks down and manual editing is necessary. Easy cases for manual editing are when the natural curvature of the skull is present and the gaps are small. Unfortunately, severe cases with large gaps and no a priori information potentially lead to large errors. Average processing time for the manual method is two hours, including threshold estimation. Average runtime including all steps of the process is 12 minutes on a fast consumer desktop computer (Intel i7 3.6@4.2 GHz processor with 16 GB ram) running Matlab. Figure 4 illustrates the two methods on example slices. Looking at the transversal slice, the methods are consistent, while on the sagittal slice the automatic method includes part of the optic canal. Fig. 5 shows the automatically estimated volumes as a function of the manual volumes. The linear regression of the two lines has been forced through origo. For the volume measured entirely inside the cut, we get $R^{2}$-value of $99.54 \%$, with a slope of $\alpha=0.9768$ being a $2.32 \%$ underestimate. Including the tetrahedra partially inside the cut, we get and $R^{2}$-value of $99.50 \%$, with a slope of $\alpha=1.0246$ being a $2.46 \%$ overestimate. We have used the worst of the volume estimates (i.e. including the partially cut tetrahedra) to calculate volume deviation, Dice score [13] and Hausdorff distance [14].

Table 1 shows the comparison between the two segmentation models. For each patient the deviation from the manual volume, Dice coefficient and Hausdorff distance were calculated to evaluate the proposed method. While the Dice coefficient measures the volume overlap, the Hausdorff distance measures the maximum error between the two segmentations. Since the Hausdorff distance measure is sensitive to single error the $95 \%$ Hausdorff distance is also included.

Deviations in volume are very small and lie between $0 \%$ and $3.7 \%$. The Dice coefficients also show a high consistency in overlap between the methods. 


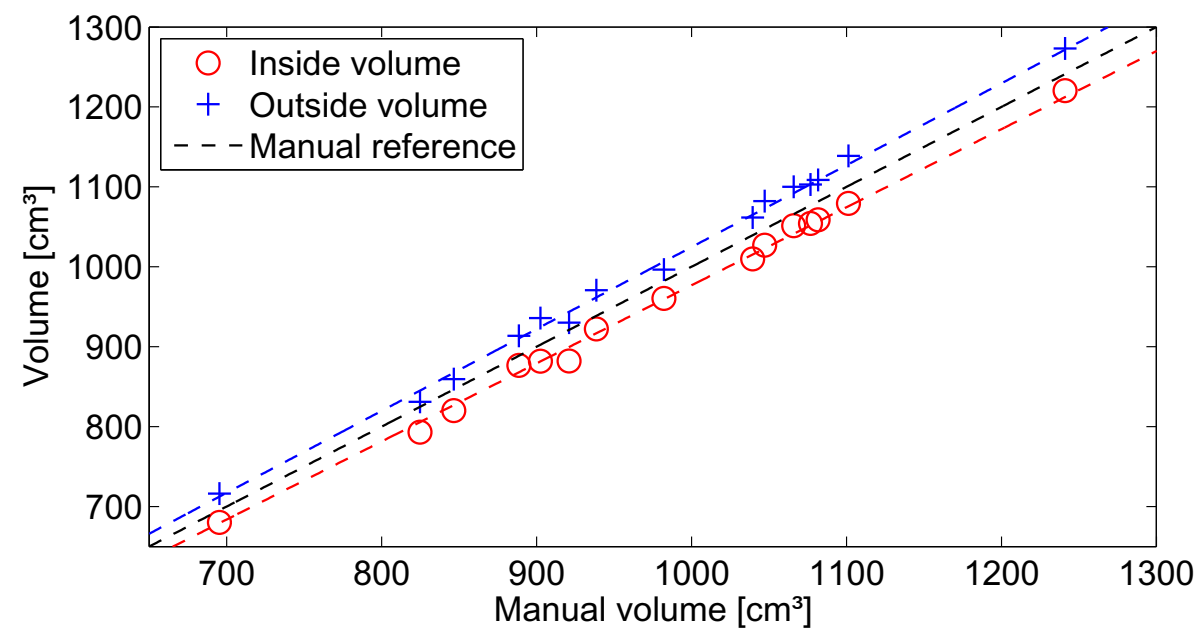

Fig. 5. Automatic volumes as a function of the corresponding manual volume including linear regression lines. Red denotes the volume with tetrahedra fully inside the segmentation, while blue also includes tetrahedra partially inside. As a reference the manual volume is shown as a black line.

Table 1. Result overview comparing the manual segmentation to the proposed automatic method. Volume deviation denotes the relative percentage-wise difference between the numeric volume estimates. Dice score measures the overlap between the volumes. Hausdorff distance assesses the maximum error. $95 \%$ Hausdorff distance is included as this measure is much less sensitive to single error.

\begin{tabular}{|c|r|r|r|l|l|l|}
\hline Patient & $\begin{array}{l}\text { Manual } \\
\text { volume } \\
{\left[\mathrm{cm}^{3}\right]}\end{array}$ & $\begin{array}{l}\text { Automatic } \\
\text { volume } \\
{\left[\mathrm{cm}^{3}\right]}\end{array}$ & $\begin{array}{l}\text { Volume } \\
\text { deviation } \\
{[\%]}\end{array}$ & $\begin{array}{l}\text { Dice } \\
\text { score } \\
{[\%]}\end{array}$ & $\begin{array}{l}\text { Hausdorff } \\
\text { distance } \\
{[\mathrm{mm}]}\end{array}$ & $\begin{array}{l}95 \% \text { Haus. } \\
\text { distance } \\
{[\mathrm{mm}]}\end{array}$ \\
\hline 1 & 846.6 & 859.5 & 1.5 & 98.87 & 7.62 & 2.22 \\
2 & 824.8 & 831.0 & 0.7 & 98.88 & 5.09 & 2.16 \\
3 & 888.6 & 913.5 & 2.8 & 98.55 & 4.94 & 1.16 \\
4 & 1076.9 & 1102.9 & 2.4 & 98.67 & 5.30 & 1.10 \\
5 & 1039.8 & 1061.7 & 2.1 & 98.71 & 6.88 & 1.72 \\
6 & 1241.1 & 1273.2 & 2.6 & 98.66 & 4.74 & 1.10 \\
7 & 982.2 & 996.5 & 1.5 & 99.00 & 9.81 & 3.65 \\
8 & 902.6 & 935.8 & 3.7 & 98.14 & 4.88 & 1.38 \\
9 & 1081.4 & 1108.8 & 2.5 & 98.67 & 4.73 & 1.12 \\
10 & 1066.1 & 1100.0 & 3.2 & 98.36 & 7.97 & 1.75 \\
11 & 1101.2 & 1138.8 & 3.4 & 98.28 & 6.05 & 1.34 \\
12 & 921.1 & 930.2 & 1.0 & 98.87 & 8.11 & 2.98 \\
13 & 695.2 & 716.2 & 3.0 & 98.39 & 6.09 & 1.89 \\
14 & 1047.5 & 1082.1 & 3.3 & 98.28 & 6.48 & 1.27 \\
15 & 938.6 & 970.8 & 3.4 & 98.29 & 5.16 & 1.22 \\
\hline
\end{tabular}


The lowest Dice score is 98.14, while the highest is 99.00. An explanation for the differences might relate to the graph cut lying slightly outside the manual border (see Fig. 4). This behavior might relate to the chosen threshold, and it would be interesting to assess the consequence of using various thresholds as well as the manual error in future work.

The Hausdorff distance, which is a conservative measure, showing the maximum error in overlaps, shows differences of up to $9.81 \mathrm{~mm}$. Examining the images, large differences between the two methods occur where the foramen magnum and optic canals are closed. Both regions are hard to segment consistently, since the spinal cord and the optic nerves are similar in intensity to that of brain matter.

\section{Concluding Remarks}

In conclusion, we have implemented an automatic, fast method for accurate estimation of the ICV in children with UCS. The method is fairly insensitive to fine tunning of parameters. There is good reason to believe that the proposed method can be used for other applications in volumetric image segmentation.

\section{References}

1. Whitwell, J.L., Crum, W.R., Watt, H.C., Fox, N.C.: Normalization of cerebral volumes by use of intracranial volume: Implications for longitudinal quantitative MR imaging. AJNR 22, 1483-1489 (2001)

2. Manjunath, K.Y.: Estimation of cranial volume - an overview of methodologies. J. Anat. Soc. India 51, 85-91 (2002)

3. Pengas, G., Pereira, J.M.S., Williams, G.B., Nestor, P.J.: Comparative reliability of total intracranial volume estimation methods and the influence of atrophy in a longitudinal semantic dementia cohort. J. Neuroimaging 19, 37-46 (2009)

4. Smith, S.M.: Fast robust automated brain extraction. Human Brain Mapping 17, 143-155 (2002)

5. Sgouros, S., Hockley, A.D., Goldin, J.H., Wake, M.J.C., Natarajan, K.: Intracranial volume change on craniosynostosis. J. Neurosurg. 91, 617-625 (1999)

6. Anderson, P.J., Netherway, D.J., Abbott, A., David, D.J.: Intracranial volume measurement of metopic craniosynostosis. J. Craniofacial Surg. 15, 1014-1016 (2004)

7. Anderson, P.J., Netherway, D.J., McGlaughlin, K., David, D.J.: Intracranial volume measurement of sagittal craniosynostosis. J. Clinical Neuroscience 14, 455-458 (2007)

8. Van Leemput, K., Maes, F., Vandermeulen, D., Suetens, P.: Automated modelbased tissue classification of MR images of the brain. IEEE Trans. on Med. Imag. 18, 897-908 (1999)

9. Li, K., Wu, X., Chen, D., Sonka, M.: Optimal surface segmentation in volumetric images-a graph-theoretic approach. IEEE Trans. on PAMI 28, 119-134 (2006)

10. Zhang, Y., Brady, M., Smith, S.: Segmentation of brain MR images through a hidden markov random field model and the expectation-maximization algorithm. IEEE Trans. on Med. Imag. 1, 45-57 (2001) 
11. Kolmogorov, V., Zabin, R.: What energy functions can be minimized via graph cuts? IEEE Trans. on PAMI 26, 147-159 (2004)

12. Shewchuk, J.: Tetrahedral mesh generation by delaunay refinement. In: Proceedings of the 14th Annual Symposium on Computational Geometry, pp. 86-95. ACM (1998)

13. Dice, L.R.: Measures of the amount of ecologic association between species. Ecology 26, 297-302 (1945)

14. Huttenlocher, D., Klanderman, G., Rucklidge, W.: Comparing images using the hausdorff distance. IEEE Trans. on PAMI 15, 850-863 (1993) 